\title{
Noise-induced resonance-like phenomena in InP crystals embedded in fluctuating electric fields
}

\section{Persano Adorno ${ }^{1}$, N Pizzolato $^{1}$ and B Spagnolo S $^{1,2,3}$}

${ }^{1}$ Dipartimento di Fisica e Chimica, Group of Interdisciplinary Theoretical Physics, Università di Palermo and CNISM-Unità di Palermo, Viale delle Scienze, edificio 18, I-90128 Palermo, Italy

${ }^{2}$ Istituto Nazionale di Fisica Nucleare, Sezione di Catania, Italy

3 Radiophysics Department, Lobachevsky State University, Nizhni Novgorod, Russia

E-mail: dominique.persanoadorno@unipa.it,nicola.pizzolato@unipa.it and bernardo.spagnolo@unipa.it

Received 20 October 2015

Accepted for publication 11 January 2016

Published 20 May 2016

Online at stacks.iop.org/JSTAT/2016/054021

doi:10.1088/1742-5468/2016/05/054021

\begin{abstract}
We explore and discuss the complex electron dynamics inside a lowdoped n-type InP bulk embedded in a sub- $\mathrm{THz}$ electric field, fluctuating for the superimposition of an external source of Gaussian correlated noise. The results presented in this study derive from numerical simulations obtained by means of a multi-valley Monte Carlo approach to simulate the nonlinear transport of electrons inside the semiconductor crystal. The electronic noise characteristics are statistically investigated by calculating the correlation function of the velocity fluctuations, its spectral density and the integrated spectral density, i.e. the total noise power, for different values of both amplitude and frequency of the driving oscillating electric field and for different correlation times of the field fluctuations. Our results show that the nonlinear response of electrons is strongly affected by the field fluctuations. In particular, crucially depending on the relationship between the correlation times of the external Gaussian noise and the timescales of complex phenomena involved in the electron dynamical behavior: (i) electrons self-organize among different valleys, giving rise to intrinsic noise suppression; (ii) this cooperative behavior causes the appearance of a resonance-like phenomenon in the noise spectra.
\end{abstract}

Keywords: stochastic particle dynamics (theory), fluctuations (theory), transport properties (theory), Boltzmann equation 


\section{Contents}

1. Introduction 2

2. The model $\quad 3$

2.1. InP structure and Monte Carlo approach. . . . . . . . . . . . . . . 3

2.2. Intrinsic electronic noise calculation and its features . . . . . . . . . . . 4

3. Numerical results and discussion 5

4. Conclusion 11

$\begin{array}{ll}\text { References } & 12\end{array}$

\section{Introduction}

The electron velocity fluctuations of hot electrons, moving inside semiconductors structures owing to static or oscillating voltages, have been extensively investigated during recent decades as the growing miniaturization process of microelectronic components causes charge carriers to be driven by increasingly intense electric fields and exhibit a highly nonlinear response [1-18].

The presence of intrinsic noise both limits the performance of semiconductor based devices and affects their sensitivity. Moreover, electronic devices are often imbedded into noisy environments that also can affect their performance.

Recently, noise-induced complex phenomena in nonlinear systems and, in particular, cooperative effects between the noise and the intrinsic interactions inside the system, have gained more and more interest [19-27]. As a matter of fact, most investigations have been oriented towards the possible positive effects of noise on nonlinear systems since several theoretical studies have revealed that, under specific conditions, the addition of external fluctuations to intrinsically noisy systems may induce an increase of the dynamical stability of the system, resulting in a less noisy response [28-40]. This counterintuitive effect is universal and, under suitable conditions, it has been found in different physical areas, ranging from the generation of spin currents [41], the enhancement of electron spin decoherence times and lengths $[42,43]$, the aggregation kinetics of Brownian particles [21, 25], the translocation dynamics of polymers [44, 45], the ultra-fast magnetization dynamics of magnetic spin systems [46, 47], to the noise redistribution in quasi 2D silicon MOS inversion layers [48].

Noise enhanced stability and the possibility of suppressing the diffusion noise by the addition of a random fluctuating contribution to the driving ciclostationary electric field, has been extensively investigated in GaAs semiconductor bulks [49-51]. Very recently preliminary results abut the possibility of tuning the dynamic electron response and obtaining noise enhanced stability phenomena in the electron transport also in low-doped n-type InP crystals have been reported in [52]. In particular, under specific conditions, we have found that the presence of time Gaussian correlated fluctuations makes the hot-carrier peak strictly resonant at the frequency of the driving periodic 
field. This fact can be seen as evidence of self-organization of the system of electrons. They are driven by the external fluctuations to oscillate among the different energy valleys exactly at the same frequency of the applied field in a cooperative way and reach a new more stable equilibrium state [52].

The aim of the present work is to deeply explore the occurrence of this stochastic resonance-like phenomenon in the noise spectra, for both (i) different frequencies of the deterministic periodic field and (ii) different intensities of the external fluctuations. Moreover, we discuss the dependence of these noise-induced positive effects on the characteristic times of the the external fluctuations. In order to simulate the nonlinear electron dynamics inside the low-doped n-type InP bulk, embedded in a sub-THz electric field, fluctuating for the presence of an external Gaussian correlated noise source, we employ a semi-classical Monte Carlo (MC) approach. Our code accounts for all the possible collisions of carriers in the crystal, the band structure details, as well as the heating effects. The electronic noise features are statistically investigated by computing the correlation function of the velocity fluctuations, its spectral density and the total noise power.

The paper is organized as follows. In section 2 we present the physical model for the $\mathrm{InP}$ band structure and the details of the MC procedure for the electron transport simulation, together with the statistical quantities used to calculate the electronic noise. In section 3 the results of our simulations are given and discussed. Final comments and conclusions are drawn in section 4.

\section{The model}

\subsection{InP structure and Monte Carlo approach}

The transport of electrons in InP bulks is simulated by using a single-particle MC algorithm which follows the standard procedure described elsewhere $[53,54]$. In this work we use a three-valley model; accordingly the conduction bands of $\mathrm{InP}$ are represented by the central $\Gamma$ valley, by four equivalent $L$-valleys and by three equivalent $X$-valleys. All most relevant scattering events of electrons in the medium, as well as the heating effects, are taken into account. The scattering rates are calculated by using the Fermi Golden Rule and the collisions are considered instantaneous. Scattering probabilities are assumed to be field-independent; therefore, the influence of the external fields is accounted for through the field-induced modifications of the electron velocities [55]. Since the sub- $\mathrm{THz}$ frequencies are below the absorption threshold, in our model we consider electrons in the conduction band as the only source of nonlinearity. Moreover, nonlinear interactions of the field with the lattice and with bound carriers are neglected. The parameters used in the present work together with the included scattering mechanisms are listed in table 1 of [56]. All the results reported here have been obtained in a steady-state regime, after that a transient time of a few ps has elapsed. All simulations run for an impurity concentration $n$ equal to $10^{13} \mathrm{~cm}^{-3}$ (non-degenerate n-type) and a lattice temperature $T_{\mathrm{L}}=77 \mathrm{~K}$ (in order to make negligible the thermal noise contribution). We assume that all donors are ionised and that the free electron concentration is equal to the doping concentration. 


\subsection{Intrinsic electronic noise calculation and its features}

In our numerical simulations the electrons inside the InP crystal are subjected to a fluctuating periodic electric field $E(t)=E \cos (\omega t)+\eta(t)$, where the deterministic term has amplitude $E$ and frequency $f=\omega / 2 \pi$. The stochastic component $\eta(t)$ is modelled by an Ornstein-Uhlenbeck (OU) process which obeys the following stochastic differential equation [57]

$$
\frac{\mathrm{d} \eta(t)}{\mathrm{d} t}=-\frac{\eta(t)}{\tau_{\mathrm{c}}}+\sqrt{\frac{2 D}{\tau_{\mathrm{c}}}} \xi(t)
$$

where $\tau_{\mathrm{c}}$ and $D$ are the correlation time and the intensity of the stochastic component, respectively [50]. The autocorrelation function of the OU process is $\left\langle\eta(t) \eta\left(t^{\prime}\right)\right\rangle=D \exp \left(-\left|t-t^{\prime}\right| / \tau_{\mathrm{c}}\right)$, and $\xi(t)$ is a Gaussian white noise with zero mean $\langle\xi(t)\rangle=0$ and $\left\langle\xi(t) \xi\left(t^{\prime}\right)\right\rangle=\delta\left(t-t^{\prime}\right)$.

The fluctuations of the electron velocity around its average value coincide with the intrinsic noise of the system. Therefore, to characterize the stochastic properties of the electron transport, we statistically analyze the velocity autocorrelation function and the mean spectral density of the velocity fluctuations. Since our sample is driven by a periodic electric field, in order to eliminate any regular contribution and describe only the fluctuating part of $v(t)$, we exploit a two-time symmetric electron velocity autocorrelation function [6]

$$
C_{\delta v \delta v}(t, \tau)=\langle v(t-\tau / 2) v(t+\tau / 2)\rangle-\langle v(t-\tau / 2)\rangle\langle v(t+\tau / 2)\rangle
$$

in which $\tau$ is the correlation time and the average is computed over a sequence of equivalent time instants $t=s+m T$, with s belonging to the time interval $[0, T]$ ( $T$ is the field period) and $m$ is an integer. In the calculation of the autocorrelation function we have considered $10^{3}$ possible initial values of $s$, and a total number of $m \sim 10^{6}$ equivalent time instants. By averaging over the whole set of values of $t$ within the period $T$, the velocity autocorrelation function becomes

$$
C_{\delta v \delta v}(\tau)=\frac{1}{T} \int_{0}^{T} C_{\delta v \delta v}(t, \tau) \mathrm{d} t
$$

The spectral density of the electron velocity fluctuations, related to the correlation function through the Wiener-Kintchine theorem, is then calculated as the Fourier transform of $C_{\delta v \delta v}(\tau)$. We emphasize that, although each single excitation is not periodic because of the presence of the Gaussian correlated component, our process exhibits cyclostationarity since its average statistical properties vary cyclically with time, i.e. $\langle E(t+n T)\rangle=\langle E(t)\rangle ;\langle v(t+n T)\rangle=\langle v(t)\rangle$, where the brackets $\langle\ldots\rangle$ stand for the average over an ensemble of different realizations of $E(t)$ and $v(t)$ [51]. Therefore, in order to calculate the noise features, we make use of the two-time symmetric correlation function described above (equations (2) and (3)).

In the case of application of a static electric field, if its amplitude is less than the threshold field needed to allow intervalley transitions (known as Gunn field, $E_{\mathrm{G}}=$ $9.7 \mathrm{kV} \mathrm{cm}^{-1}$ in $\mathrm{InP}$ ), the noise spectral density shows a Lorentzian shape with a cut-off frequency related to the mean scattering frequency of the electron system [1]. The intrinsic electronic noise, knows as diffusion noise, is mainly due to the fluctuations in 
the $k$-space, originating from the collisions of electrons with phonons, impurities, etc [58]. If the field strength becomes greater than the Gunn field, it is no longer possible to disregard the InP band structure. Consequently, the stochastic electron transitions in momentum space among valleys, characterized by different dynamical properties, must be correctly taken into account. In this case, in addition to the diffusive term, the intrinsic electronic noise contains an extra contribution, due to fluctuations in the number of carriers in the valleys, known as partition noise [1]. The spectral density of the partition noise assumes a Lorentzian shape characterized by the average velocities in the $\Gamma$ valley and in the $L$ - and $X$-valleys and by a characteristic intervalley exchange time known as $\tau_{0}$ (see equations (8) and (9) in [11] for details). The partition noise is characterized by a pronounced peak in the spectral density at frequency $\nu_{0}=1 / \tau_{0}$, which can be considered as a natural transition frequency of the system between the different energy valleys. The height of the peak depends on the population of the different valleys and reaches a maximum value when the populations are nearly the same [11]. In particular $\nu_{0}$ increases with the increasing strength of the external field since the growing of available energy of the carriers causes the rising of the characteristic intervalley exchange rate. Further increasing the strength of the applied field, the population of the $\Gamma$ valley becomes negligible with respect to that of the higher energy valleys and the main noise source becomes the diffusion in the $L$ - and $X$-valleys, the mean scattering frequency raises and therefore we observe an increasing of the cut-off frequency and a decreasing in the value of the spectral density in the low-frequency range.

When the driving electric field is oscillating, the characteristic transition frequency of the system $\nu_{f}$ may depend also on the frequency $f$ of the forcing field. Thus, in cyclostationary regime, depending on the ratio between the frequency $f$ of the forcing field and the transition frequency of the electron system, different dynamical responses are expected. If the electrons are subjected to a small-amplitude electric field, independently from the excitation frequency, a Lorentzian diffusion noise is always expected, because all carriers are located in the $\Gamma$ valley. For low-frequency fields $\left(f<\nu_{0}\right.$, where $\nu_{0}$ is the natural transition frequency calculated in the static case), a noise behavior similar to that found in the static field case is expected, i.e. a spectrum exhibiting a Lorentzian shape for weak field $\left(E<E_{\mathrm{G}}\right)$ and a hot-carrier peak for $E>E_{\mathrm{G}}$. In this case, the frequency of the hot-carrier peak is not related with the excitation frequency. If the driving field frequency $f$ becomes greater than $\nu_{0}$, the system becomes forced to exchange electrons among the valleys at a frequency close to that of the external field, $\nu_{f}$ shifts towards higher values closer to the applied field frequency, making the velocity fluctuations time-correlated. In this forced response regime, the spectral density can significantly differ from the typical partition noise behavior [11].

\section{Numerical results and discussion}

In [52] the spectral density of the electron velocity fluctuations along the direction of application of a static electric field has been investigated. In particular, with the same values of lattice temperature and doping concentration used in the present work, the natural transition frequency $\nu_{0}$ of the system between the different energy valleys has been found at about $\nu \approx 500 \mathrm{GHz}[52]$. 
In panels (a)-(d) of figure 1 we show the spectral density of the electron velocity fluctuations along the direction of a cyclostationary applied field, for different values of the amplitude of the driving field, at four frequencies (namely, $f=100,500,800$, $1000 \mathrm{GHz}$ ) and a lattice temperature $T_{\mathrm{L}}=77 \mathrm{~K}$. In all panels dashed lines show the spectra without any external electric field applied. As expected, the spectral density computed at the lowest frequency ( $f=100 \mathrm{GHz}$-panel (a)) is analogous to that obtained in the static field case (see for comparison figure 1 of [52]), except for an increase in the low-frequency region. The spectra obtained at field amplitudes greater than the threshold field for intervalley transitions, show the typical shape characterized by the presence of a hot-carrier peak at a frequency close to the natural frequency found in the static case $(\nu \approx 500 \mathrm{GHz})$. A very similar noise spectra has been found if the frequency of the oscillating field varies in the range $f=200-400 \mathrm{GHz}$.

Conversely, when the external frequency $f$ approaches to or overcomes the natural frequency of transitions among the valleys (as for $f=500,800$ and $1000 \mathrm{GHz}$-panels (b)-(d)), we may observe different behaviors. In particular, for the weakest field intensity $\left(E=10 \mathrm{kV} \mathrm{cm}^{-1}\right)$ the intrinsic noise results essentially to be due to the diffusion process, and, as expected, the spectral density shows a typical Lorentzian shape.

By increasing the field amplitude up to values strong enough to trigger intervalley transitions, the noise spectra significantly change, showing a prominent enhancement of the density in the low-frequency region and a forced hot-carrier peak, located closely to the frequency of the external field. The occurrence of this forced regime may be related to the ratio $E / f$, which can be considered a discriminating parameter quantifying the change of carrier momentum during a free flight. In particular, if $E / f>0.025 \mathrm{kV}$ $\mathrm{cm}^{-1} \mathrm{GHz}^{-1}$, the external field oscillations drive the intervalley tranfers and the hotcarrier peak moves close to the frequency of the driving field, otherwise the system is in the usual hot-carrier regime. Further increasing the field amplitude, the electron system moves away from the forced transfer regime and the spectral density of the velocity fluctuations exhibits a relevant noise suppression in the low- frequency region. At even higher voltages, since the electrons spend most of the time in the upper valleys, the main source of intrinsic noise becomes again diffusion; therefore the spectral density assumes again a Lorentzian shape. Moreover, since in the $L$ - and $X$-valleys the diffusion coefficient reduces its value with respect of that of the $\Gamma$ valley, the spectral density further reduces.

In a previous paper [52] it has been shown that, strictly depending on the timescales characterizing the external noise source, the superimposition of Gaussian time-correlated fluctuations to a forcing electric field oscillating at $f=500 \mathrm{GHz}$ makes the forced peak strictly resonant at the frequency of the driving periodic field.

Here the occurrence and dependence of this stochastic resonance-like phenomenon has been investigated for different frequencies of the deterministic periodic field, different amplitudes of the external fluctuations and different noise correlation times. Panels (a) and (b) of figures 2-4 show how the spectral density of the electron velocity fluctuations modifies at about the frequency of the driving field in the presence of Gaussian correlated fluctuations, for different values of the noise correlation time $\tau_{\mathrm{c}}$, superimposed to a field oscillating at $f=500,800$ and $1000 \mathrm{GHz}$, respectively. In particular, the spectra drawn in panels (a) have been computed with $D^{1 / 2}$ equal to $20 \%$ of the value of the field amplitude; those in panels (b) with a $D^{1 / 2}$ equal to $40 \%$ of $E$. The amplitude of the 
Noise-induced resonance-like phenomena in InP crystals
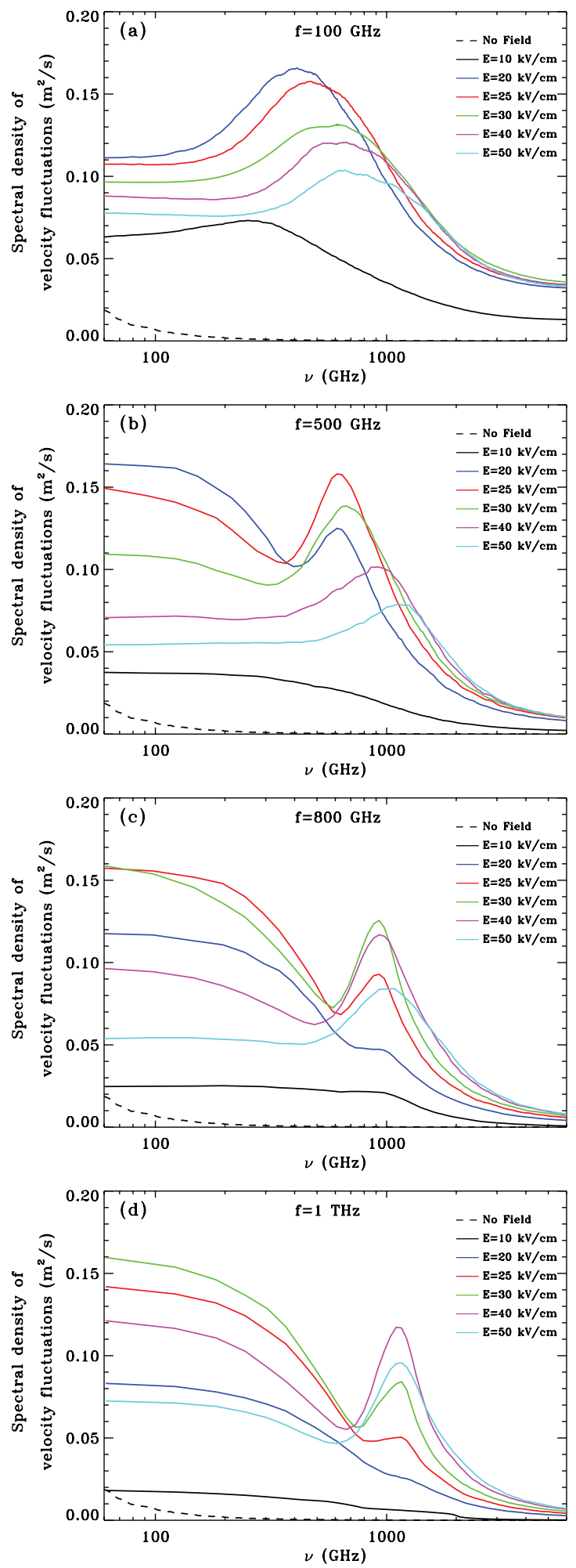

Figure 1. Spectral density of the electron velocity fluctuations, along the direction of the ac applied voltage, obtained in the case of a driving periodic electric field having frequency $f$ : (a) $100 \mathrm{GHz}$, (b) $500 \mathrm{GHz}$, (c) $800 \mathrm{GHz}$, (d) $1 \mathrm{THz}$, for different values of its amplitude; dashed lines show the spectra without any external electric field applied. $T_{\mathrm{L}}=77 \mathrm{~K}$. 
Noise-induced resonance-like phenomena in InP crystals
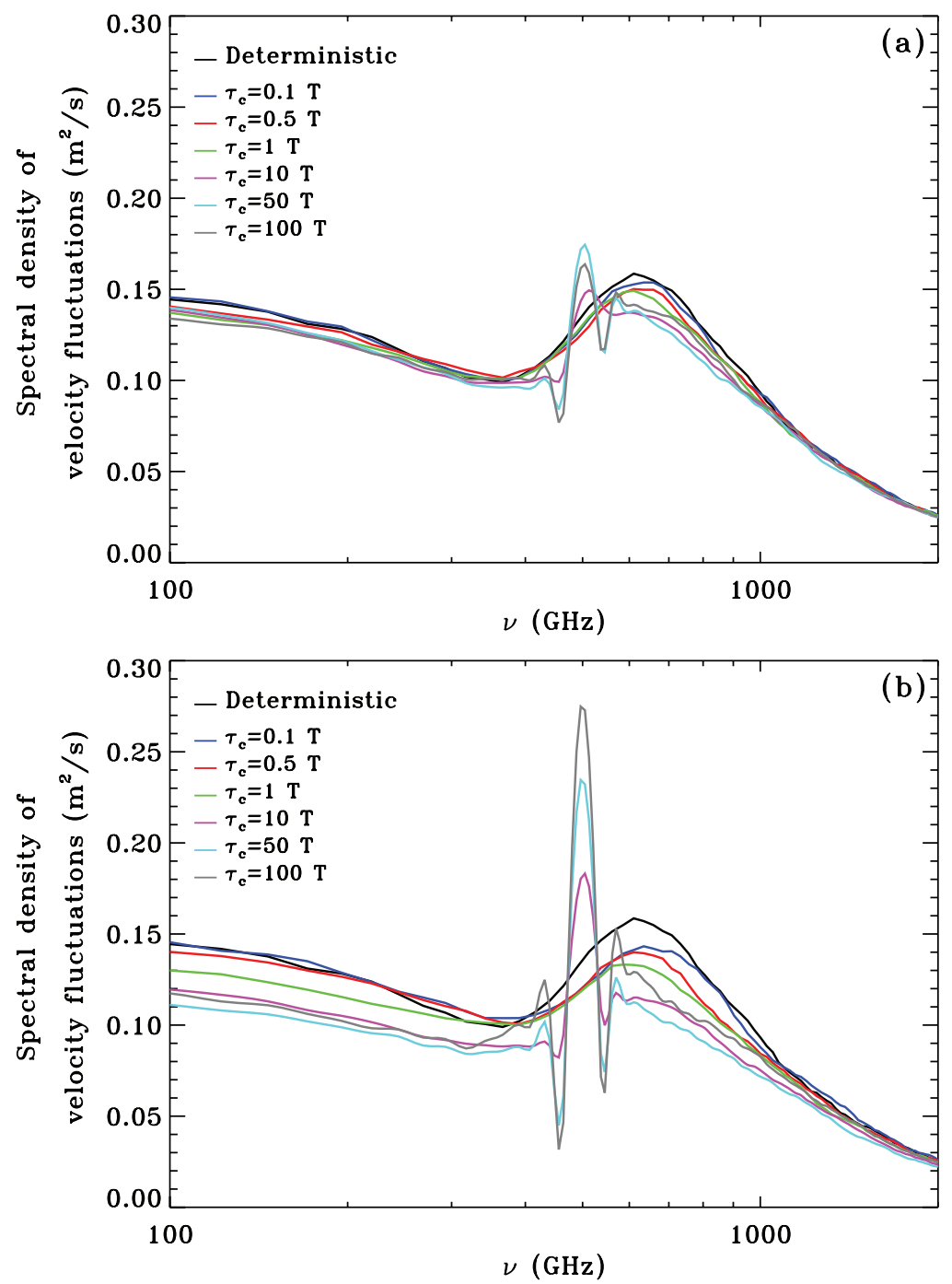

Figure 2. Spectral density of the electron velocity fluctuations, along the direction of the fluctuating applied electric field, obtained when Gaussian time-correlated fluctuations are superimposed to the forcing oscillating voltage, for different values of the noise correlation time $\tau_{\mathrm{c}}$. The other parameter values are: $E_{f}=25 \mathrm{kV} \mathrm{cm}^{-1}$, $f=500 \mathrm{GHz}, T_{\mathrm{L}}=77 \mathrm{~K}$, (a) $D^{1 / 2}=5 \mathrm{kV} \mathrm{cm}^{-1}$, (b) $D^{1 / 2}=10 \mathrm{kV} \mathrm{cm}^{-1}$.

deterministic periodic field has been chosen in order to allow the intervalley transitions and to maximize the forced response regime, namely $E_{f}=25 \mathrm{kV} \mathrm{cm}^{-1}$ at $f=500 \mathrm{GHz}$, $E_{f}=30 \mathrm{kV} \mathrm{cm}^{-1}$ at $f=800 \mathrm{GHz}, E_{f}=40 \mathrm{kV} \mathrm{cm}^{-1}$ at $f=1 \mathrm{THz}$. In all cases, we find that the spectrum calculated in the absence of external fluctuations is modified in a way that strongly depends on the relationship among the period $T$ of the ciclostationary electric field and the correlation time $\tau_{\mathrm{c}}$ of the stochastic OU process. At short correlation times $\left(\tau_{\mathrm{c}}=0.1,0.5 \mathrm{~T}\right)$, the changes observed in the height of the peak are due to the circumstance that, because of the presence of the external time-correlated fluctuations, the effective electric field experienced by the electrons changes, implying a modification in the number of intervalley transfers with respect to the case in which the external noise is negligible. Obviously, the changes are most evident in all panels (b), where the amplitude of the stochastic component is set equal to $40 \%$ of the value of the field amplitude. 
Noise-induced resonance-like phenomena in InP crystals
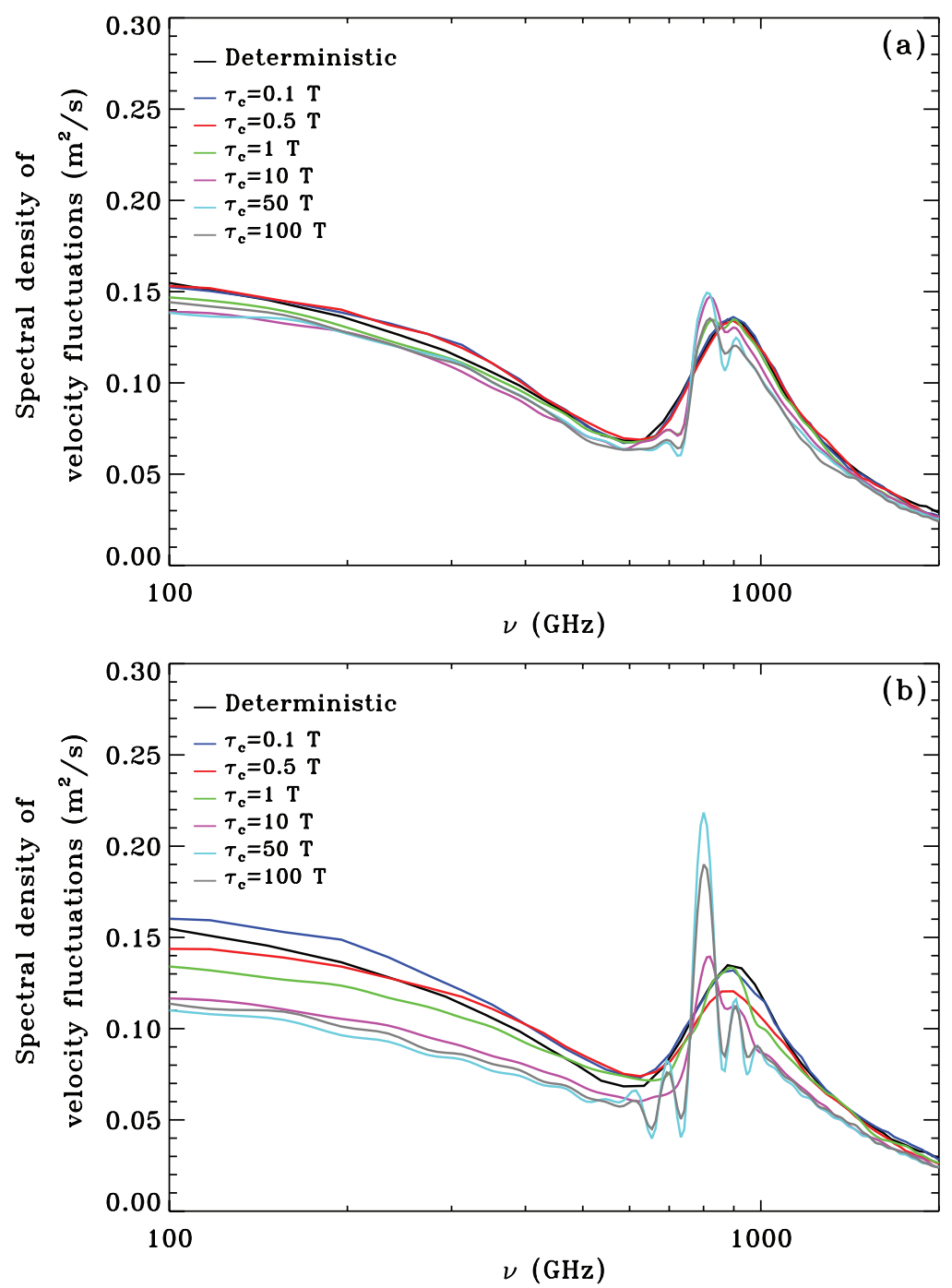

Figure 3. Spectral density of the electron velocity fluctuations, along the direction of the fluctuating applied electric field, obtained when Gaussian time-correlated fluctuations are superimposed to the forcing oscillating voltage, for different values of the noise correlation time $\tau_{\mathrm{c}}$. The other parameter values are: $E_{f}=30 \mathrm{kV} \mathrm{cm}^{-1}$, $f=800 \mathrm{GHz}, T_{\mathrm{L}}=77 \mathrm{~K}$, (a) $D^{1 / 2}=6 \mathrm{kV} \mathrm{cm}^{-1}$, (b) $D^{1 / 2}=12 \mathrm{kV} \mathrm{cm}^{-1}$.

The spectra obtained at $\tau_{\mathrm{c}}=1 \mathrm{~T}$ (green line in all panels) show different features, crucially depending on the frequency of the driving field. In particular, at $f=500$ $\mathrm{GHz}$ (figure 2), the spectra are only slightly modified, as described above for the cases of $\tau_{\mathrm{c}}=0.1,0.5 \mathrm{~T}$. On the other hand, the spectra obtained at $f=800 \mathrm{GHz}$ (figure 3) and $f=1 \mathrm{THz}$ (figure 4) are characterized by different shapes, showing a narrower hot-carrier peak not centered at the frequency of the driving periodic field. This finding highlights the beginning of a transition towards a different noise-induced system response regime.

The most interesting effect has been found for noise correlation times equal to or greater than $10 \mathrm{~T}$. Indeed in such cases, for all the three investigated frequencies, the superimposition of Gaussian time-correlated fluctuations modifies entirely the noise spectra. The hot-carrier peak narrows and becomes strictly resonant at the frequency of 
Noise-induced resonance-like phenomena in InP crystals
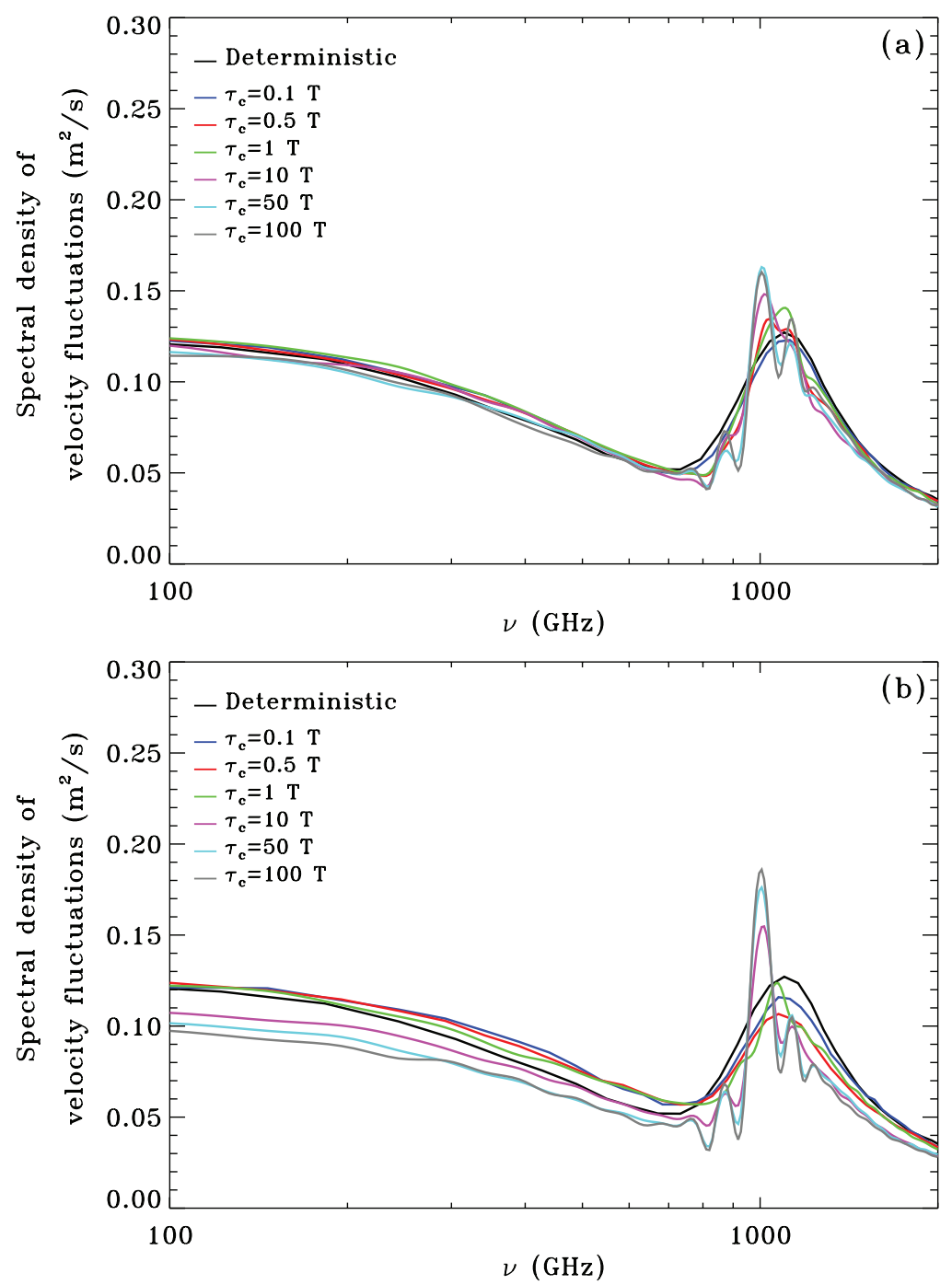

Figure 4. Spectral density of the electron velocity fluctuations, along the direction of the fluctuating applied electric field, obtained when Gaussian time-correlated fluctuations are superimposed to the forcing oscillating voltage, for different values of the noise correlation time $\tau_{\mathrm{c}}$. The other parameter values are: $E_{f}=40 \mathrm{kV} \mathrm{cm}^{-1}$, $f=1 \mathrm{THz}, T_{\mathrm{L}}=77 \mathrm{~K}$, (a) $D^{1 / 2}=8 \mathrm{kV} \mathrm{cm}^{-1}$, (b) $D^{1 / 2}=16 \mathrm{kV} \mathrm{cm}^{-1}$.

the driving periodic field. Moreover, (i) harmonic peaks appear, whose height increases with the increasing of the noise correlation time and (ii) the spectral density in the lowfrequency range decreases. This resonance-like phenomenon could be an evidence that electrons transfer among the different energy valleys exactly at the same frequency of the applied field in a cooperative way, reaching a new more stable equilibrium state. This can be interpreted as an example of self-organization of the electron system promoted by the presence of external fluctuations having suitable correlation times.

In order to investigate the occurrence of a noise-induced intrinsic noise suppression, we have calculated the Integrated Spectral Density (ISD), which corresponds to the variance of the electron velocity. We show in figure 5 our numerical outcomes of ISD, normalized to the value obtained in the deterministic case, as a function of the ratio between the noise correlation time $\tau_{\mathrm{c}}$ and the period of the driving field $T$, for all the 
Noise-induced resonance-like phenomena in InP crystals

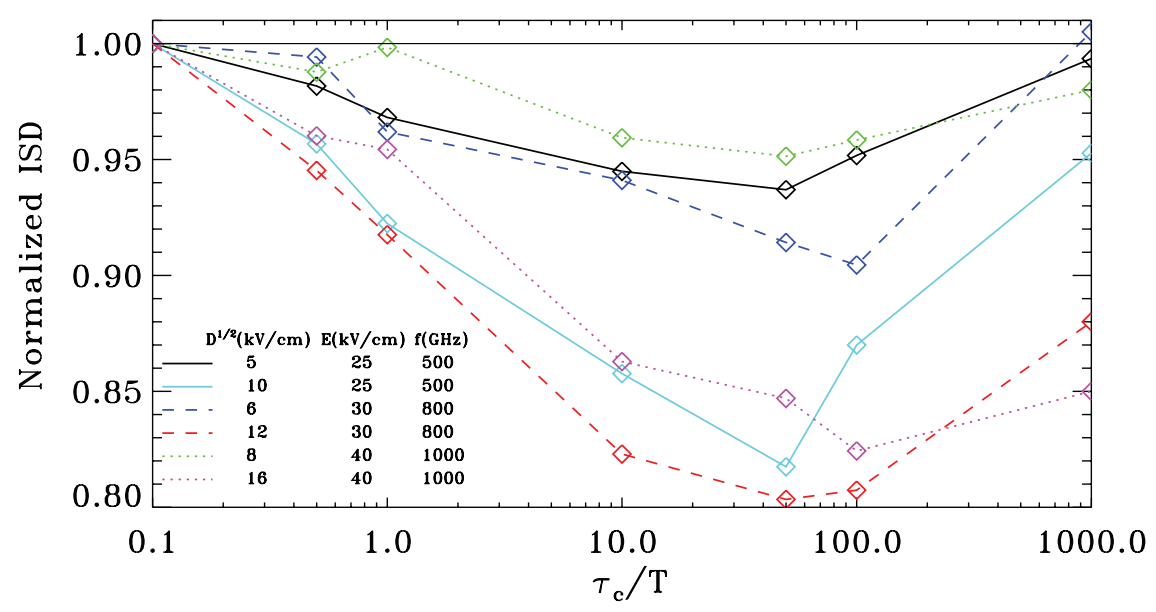

Figure 5. ISD of the electron velocity fluctuations (normalized to the value obtained in the deterministic case) as a function of the ratio between the noise correlation time $\tau_{\mathrm{c}}$ and the period $T$ of the oscillating driving field. $T_{\mathrm{L}}=77 \mathrm{~K}$.

three investigated frequencies and the two values of noise intensity. When the Gaussian correlated contribution is added to the periodic electric field it is possible to observe a clear reduction of the ISD, i.e. a less noisy response. This suppression enhances with the increase of the amplitude of the external fluctuations, up to about 20\%. Moreover, the total noise power reduces for values of noise correlation time up to $50 \mathrm{~T}$. This reduction, previously observed in GaAs bulks [50], could be ascribed to a noise enhanced stability, arising from the the cooperative interplay between the fluctuating electric field and the intrinsic fluctuations in the complex dynamics of electron system inside the semiconductor crystal. Our findings suggest that the external noise constructively force the electrons to oscillate at exactly the same frequency of the driving field, performing a more ordered dynamics confirmed by a lower total noise power. As discussed in [33], the noise suppression may be due to the fact that the fluctuating electric field forces the electrons to visit regions of the momentum space characterized by a smaller variance with respect to the case of zero external fluctuations.

\section{Conclusion}

In the present work we report the results from a many-valley Monte Carlo investigation of the electron nonlinear transport in low-doped n-type InP crystals operating under a fluctuating sub- $\mathrm{THz}$ electric field, at three different frequencies of the driving field. The electronic noise properties were statistically investigated by computing the spectral density of the electron velocity fluctuations and the total noise power. The noise spectra obtained with a deterministic oscillating driving field exhibit some interesting peculiarities depending on both the amplitude and the frequency of the applied electric field. In particular, if the amplitude of the driving field is strong enough to switch on intervalley transitions from the lower valley $(\Gamma)$ to the upper valleys ( $L$ and $X$ ), the noise spectra show a peak shifting towards higher frequencies and a significant noise enhancement in the low-frequency region. 
This behaviour can be modified if the driving periodic electric field is affected by Gaussian time-correlated fluctuations, where the noise amplitude, the ratio between the correlation time $\tau_{\mathrm{c}}$ of the OU stochastic process and the period $T$ of the oscillating electric field appear to be the crucial quantities to tune for studying this effect. First, a less noisy response is observed in the presence of a fluctuating periodic electric field. This noise-induced effect suggests that, under suitable timescales, the complex dynamics of electrons in the crystal benefits from the cooperative interplay between the fluctuating electric field and the intrinsic fluctuations of the system itself. Then, for noise correlation times equal or greater than $10 \mathrm{~T}$, a resonance-like phenomenon in the noise spectra is found. The addition of the time-correlated fluctuations affects the electron dynamics, forcing a cooperative nonlinear behaviour of carriers which self-organize. They, driven by the external fluctuations, oscillate among the different energy valleys exactly at the same frequency of the applied field and reach a less noisy equilibrium state. This means that time-correlated fluctuations externally superimposed to a driving electric field could play an important role on controlling and tuning the electronic noise in InP based electronic devices. In fact, by using noise sources with suitable characteristic times, it could be possible to select the favorable conditions for the best operating of InP electronic structures, for example in terms of an increase of the signal to noise ratio.

The results described in this paper leave several open problems on the intrinsic physical mechanism beyond these cooperative effects. Further studies are needed to deeply investigate why, under suitable conditions, the external fluctuations constructively contribute to force the electrons to perform a more ordered dynamics. Furthermore, it could be very interesting to study these noise-induced effects also for (i) different typologies of the external fluctuations, such as symmetric dichotomous noise, colored noise, etc, and in (ii) different semiconductors structures in order to investigate the role played by the band structure.

\section{References}

[1] Nougier J P 1994 IEEE Trans. Electron Devices 412034

[2] Kogan Sh 1996 Electronic Noise and Fluctuations in Solids (Cambridge: Cambridge University Press) chapter 3

[3] Matulionis A, Raguotis R and Katilius R 1997 Phys. Rev. B 562052

[4] Hartnagel H L, Katilius R and Matulionis A 2001 Microwave Noise in Semiconductor Devices (New York: Wiley)

[5] Shiktorov P et al 2002 Phys. Status Solidi a 190271

[6] Gonzalez T et al 2003 Proc. SPIE 5113252

[7] Shiktorov P, Starikov E, Gruzinskis V, Reggiani L, Varani L, Vaissiere J C, Perez S and Gonzalez T 2004 Semicond. Sci. Technol. 19 S170

[8] Dyakonov M and Shur M S 2005 Appl. Phys. Lett. 87111501

[9] Pankratov E L and Spagnolo B 2005 Eur. Phys. J. B 4615

[10] Matulionis A 2007 Lith. J. Phys. 47297

[11] Persano Adorno D, Capizzo M C and Zarcone M 2008 Fluct. Noise Lett. 8 L11

[12] Millithaler J F, Reggiani L, Pousset J, Sabatini G, Varani L, Palermo C, Mateos J, Gonzlez T, Perez S and Pardo D 2008 J. Phys.: Condens. Matter 20384210

[13] Millithaler J F, Reggiani L, Pousset J, Varani L, Palermo C, Knap W, Mateos J, Gonzalez T, Perez S and Pardo D 2008 Appl. Phys. Lett. 92042113

[14] Varani L et al 2006 J. Comput. Electron. 571

[15] Persano Adorno D, Capizzo M C and Pizzolato N 2008 Acta Phys. Pol. A 113979

[16] Millithaler J F et al 2009 J. Stat. Mech. P02030

[17] Rengel R and Martin M J 2013 J. Appl. Phys. 114143702 
Noise-induced resonance-like phenomena in InP crystals

[18] Asmontas S, Raguotis R and Bumeliene S 2014 Solid State Sci. 38156

[19] Shuda I A, Borysov S S and Olemskoi A I 2009 Phys. Scr. 79065001

[20] Li J 2010 Physica A 3897

[21] Ghosh P, Chattopadhyay S and Chaudhuri J R 2012 Chem. Phys. 40248

[22] Nakada K, Yakata S and Kimura T 2012 IEEE Trans. Magn. 484558

[23] Valenti D, Denaro G, Persano Adorno D, Pizzolato N, Zammito S and Spagnolo B 2012 Cent. Eur. J. Phys. 10560

[24] Wang B, Sun Y Q and Tang X D 2013 Chin. Phys. B 22010501

[25] Sen M K, Ray S, Baura A and Bag B C 2013 Chem. Phys. Lett. 559117

[26] Gottwald G A and Harlim J 2013 Proc. R. Soc. A 46920130096

[27] Sonnenschein B, Peron T K, Rodrigues F A, Kurths J and Schimansky-Geier L 2015 Phys. Rev. E 91062910

[28] Pikovsky A S and Kurths J 1997 Phys. Rev. Lett. 78775

[29] Landa P S 2000 Phys. Rep. 3231

[30] Vilar J M and Rubi J M 2001 Phys. Rev. Lett. 86950

[31] Seol Y, Visscher K and Walton D B 2004 Phys. Rev. Lett. 93160602

[32] Spagnolo B, Agudov N V and Dubkov A A 2004 Acta Phys. Pol. B 351419

[33] Walton D B and Visscher K 2004 Phys. Rev. E 69051110

[34] Dubkov A A, Agudov N V and Spagnolo B 2004 Phys. Rev. E 69061103

[35] Fiasconaro A and Spagnolo B 2009 Phys. Rev. E 80041110

[36] Romanczuk P, Bär M, Ebeling W, Lindner B and Schimansky-Geier L 2012 Eur. Phys. J. 2021

[37] Martens S, Straube A V, Schmid G, Schimansky-Geier L and Hänggi P 2013 Phys. Rev. Lett. 110010601

[38] Sonnenschein B and Schimansky-Geier L 2012 Phys. Rev. E 85051116

[39] Kromer J A, Pinto R D, Lindner B and Schimansky-Geier L 2014 Europhys. Lett. 10820007

[40] Sonnenschein B, Peron T K, Rodrigues F A, Kurths J and Schimansky-Geier L 2014 Eur. Phys. J. B 87182

[41] Dugaev V K, Inglot M, Sherman E Ya and Barnas J 2012 J. Magn. Magn. Mater. 3243573

[42] Spezia S, Persano Adorno D, Pizzolato N and Spagnolo B 2012 J. Phys.: Condens. Matter 24052204

Spezia S, Persano Adorno D, Pizzolato N and Spagnolo B 2012 Acta Phys. Pol. B 431191

[43] Spezia S, Persano Adorno D, Pizzolato N and Spagnolo B 2013 Europhys. Lett. 10447011

[44] Pizzolato N, Fiasconaro A, Persano Adorno D and Spagnolo B 2010 Phys. Biol. 7034001

[45] Pizzolato N, Fiasconaro A, Persano Adorno D and Spagnolo B 2013 J. Chem. Phys. 138054902

[46] Atxitia U, Chubykalo-Fesenko O, Chantrell R W, Nowak U and Rebei A 2009 Phys. Rev. Lett. 102057203

[47] Trapanese M 2009 J. Appl. Phys. 10507 D313

[48] Lodato M A, Persano Adorno D, Pizzolato N and Spagnolo B 2013 Acta Phys. Pol. B 441163

[49] Persano Adorno D, Pizzolato N and Spagnolo B 2008 Acta Phys. Pol. A 113985

[50] Persano Adorno D, Pizzolato N and Spagnolo B 2009 J. Stat. Mech. P01039

[51] Persano Adorno D, Pizzolato N, Valenti D and Spagnolo B 2012 Rep. Math. Phys. 70171

[52] Persano Adorno D, Pizzolato N, Alaimo P, Spagnolo B and Di Paola B 2015 Chaos Soliton Fractals 81425

[53] Persano Adorno D, Zarcone M and Ferrante G 2000 Laser Phys. 10310

[54] Persano Adorno D, Zarcone M and Ferrante G 2001 Laser Part. Beams 1981

Persano Adorno D, Zarcone M and Ferrante G 2001 Laser Phys. 11291

[55] Persano Adorno D 2010 Laser Phys. 201061

[56] Persano Adorno D, Pizzolato N and Fazio C 2015 Eur. J. Phys. 36055017

[57] Gardiner C 2009 Stochastic Methods: A Handbook for the Natural and Social Sciences (Springer Series in Synergetics) 4th edn (Berlin: Springer)

[58] Shockley W, Copeland J A and James R P 1966 Quantum Theory of Atoms, Molecules and the Solid State (New York: Academic) 